\title{
Aktuelle Aspekte der Diagnostik und Therapie der Oberflächen- thrombose des Beines
}

\section{Stücker; S. Reich-Schupke}

Venenzentrum der Dermatologischen und Gefäßchirurgischen Kliniken, Kliniken der Ruhr-Universität Bochum, Bochum

\author{
Schlüsselwörter \\ Oberflächenthrombose, Heparin, Kompressi- \\ on, Fondaparinux, Rivaroxaban, Duplexsono- \\ grafie
}

\section{Zusammenfassung}

Der derzeit aktuelle Terminus Oberflächenthrombose spiegelt besser als der alte Begriff Thrombophlebitis die Ernsthaftigkeit des Krankheitsbildes wider, welches bei etwa 25\% der Patienten mit weitere thromboembolischen Komplikationen wie tiefen (Bein)Venenthrombosen und Lungenembolien einhergeht. Treten die Oberflächenthrombosen in Varizen auf, sollten diese nach Abheilung der akuten OVT saniert werden. Oberflächenthrombosen abseits der Varizen treten gehäuft bei malignen Grunderkrankungen, Thrombophilie und anderen Risikofaktoren der tiefen Beinvenenthrombose auf. Obwohl die Diagnose in der Regel klinisch gestellt werden kann, ist die Duplexsonographie unverzichtbar, um die Ausdehnung des Thrombus und ein mögliches Übergreifen des Thrombus in das tiefe Beinvenensystem zu erfassen. Dabei ist das komplette Venensystem beider Beine vollständig zu sonografieren, da der entscheidende Faktor für die Oberflächenthrombose die Hyperkoagulabilität ist und daher begleitende tiefe Venenthrombosen durchaus auch auf der Gegenseite auftreten können. Die Therapie der Oberflächenthrombose ist gestaffelt nach den betroffenen $\mathrm{Ge}$ fäßen: 1) Bei kleinkalibrigen Astthrombosen sind Kühlung, Kompressionstherapie, nicht steroidale Antiphlogistika sowie eine Stichinzision mit Thrombusexpression angezeigt. 2) Bei Oberflächenthrombosen der Saphenavenen und großkalibriger Varizenäste über $5 \mathrm{~cm}$ Länge wird eine Antikoagulation in Prophylaxedosierung über 4-6 Wochen empfohlen, begleitet durch eine Kompressionstherapie über 3 Monate. Bei Risikofaktoren wie Krebsleiden, Autoimmunerkrankungen oder Befall nicht variköser Venen kommt es nicht selten erst nach Absetzen einer 6wöchigen Antikoagulation zu thromboembolischen Komplikationen, so dass bei diesen Patienten entsprechende Instruktionen sinnvoll sind. 3) Reicht der Thrombus näher als $3 \mathrm{~cm}$ an das tiefe Venensystem heran bzw. greift er auf die tiefen Venen über, wird wie bei einer tiefen Beinvenenthrombose therapiert.

\section{Keywords}

Superficial venous thrombosis, heparin, compression, fondaparinux, rivaroxaban, duplex

Current aspects of diagnosis and treatment of surface thrombosis of the leg

Phlebologie 2018; 47: 329-333

https://doi.org/10.12687/phleb2434-6-2018

Eingegangen: 26. Juni 2018

Angenommen: 28. Juni 2018

English version available at: www.thieme.de/phlebo

\section{Summary}

The term "superficial venous thrombosis" (SVT) is more suitable to characterize the impact of the underlying disease instead of the old term "thrombophlebitis", since $25 \%$ of the patients have additional thrombembolic complications as a deep venous thrombosis or pulmonary embolism. If SVT is found in varicose veins, these veins should be therapied after the healing of the acute thrombosis. SVT independent of varicose veins are often seen in patients with malignancies, thrombophilia and other risk factors oft he deep vein thrombosis. Although the diagnosis of SVT could be made by clinical findings a creful duplex is essential to detect the extend of the thrombus and the exact location - perhaps with progress into the deep venous system. The complete venous system of both legs should be examined as the main reason for the SVT is hypercoagulability. Therefore, concomitant deep venous thrombosis can be detected on the same but also on the other leg. The therapy of SVT depends on the affected vein: 1) In small tributary veins cooling, compression therapy and nonsteroidal antiinflammatory drugs as well as a small inzision and expression of the thrombus are sufficient. 2) In SVT of saphenous veins and larger tributaries with a length of $5 \mathrm{~cm}$ or more, anticoagulation in prophylactic dose for 4-6 weeks and compression treatment for 3 months is recommended. In patients with risk factors like cancer, autoimmune disease or SVT in non-varicose veins, thromboembolic complications are often seen after the end of the 6-weeks anticoagulation. In these patients special instructions are helpful. 3) A SVT nearby $(<3 \mathrm{~cm})$ the crossing to the deep venous system or with extend into the deep venous system should be treated like a deep venous thrombosis. 


\section{Thrombophlebitis/Venen- entzündung oder Oberflächenthrombose?}

In Deutschland wurde lange der Begriff Thrombophlebitis bzw. Venenentzündung bevorzugt, um gegenüber dem Patienten eine bessere Abgrenzung gegenüber der tiefen Beinvenenthrombose zu haben. International wie auch national hat sich dies jedoch geändert. Derzeit wird auch in Deutschland der Begriff Oberflächenthrombose präferiert. Grund hierfür ist, dass die gewichtete mittlere Prävalenz der tiefen Beinvenenthrombose zum Zeitpunkt der Oberflächenthrombose bei $18,1 \%$ liegt und die der Lungenembolie bei $6,9 \%$, wie eine Meta-Analyse aus 21 Studien mit insgesamt 4358 Patienten zur Prävalenz der tiefen Beinvenenthrombose und einer Analyse aus 11 Studien mit 2484 Patienten zur Prävalenz der Lungenembolie bei $\mathrm{Pa}$ tienten mit oberflächlicher Venenthrombose nachweisen konnte (1). Doch nicht nur im Hinblick auf die möglichen Komplikationen weiterer thromboembolischer Erkrankungen, sondern auch im Hinblick auf die korrekte Therapie sollten die Begriffe Thrombophlebitis und Venenentzündung vermieden werden, da auf diese Art und Weise die nicht seltene, aber fehlindizierte Verordnung von Antibiotika reduziert werden kann. So verordneten in einer Untersuchung in Großbritannien immerhin etwa 20\% der Ärzte Antibiotika zur Therapie der Oberflächenthrombose (2).

\section{Häufigkeit der Oberflächenthrombose}

Mit einer jährlichen Inzidenz von 0,64 Promille ist die Oberflächenthrombose eine relativ häufige Erkrankung. Dies zeigte sich bei einer prospektiven multizentrischen Studie in einer Region um St. Etienne in Frankreich über ein Jahr im Zeitraum 2011 bis 2012. Die Oberflächenthrombose war in dieser Studie bei Frauen häufiger und stieg mit zunehmendem Alter unabhängig vom Geschlecht an. Auch in dieser Studie hatte ein hoher Anteil von 24,6\% der Patienten eine begleitende tiefe Beinvenen- thrombose, 4,7\% der Patienten eine Lungenembolie (3).

Das gleichzeitige Auftreten von Oberflächenthrombosen und einer tiefen Beinvenenthrombose oder einer Lungenembolie ist in der Literatur gut und übereinstimmend dokumentiert. Interessant ist nun, dass auch in den Monaten und Jahren nach dieser Erkrankung ein erhöhtes Risiko für thromboembolische Ereignisse besteht. Immerhin 3,4\% der Patienten mit einer Oberflächenthrombose ohne begleitende tiefe Beinvenenthrombose zum Zeitpunkt der Erstdiagnose entwickeln später noch eine tiefe Beinvenenthrombose (4). Verglichen mit der Allgemeinbevölkerung war das Risiko in den ersten drei Monaten nach einer Oberflächenthrombose 71,4-fach erhöht, das Risiko einer tiefen Beinvenenthrombose sinkt in den Folgemonaten und -jahren zwar ab, liegt aber auch 5 Jahre nach der Oberflächenthrombose noch 5,1-fach höher als in der Vergleichsgruppe. Auch das Risiko einer Lungenembolie ist nach einer Oberflächenthrombose in den ersten drei Monaten 45,4-fach, nach 5 Jahren immerhin noch 2,9-fach erhöht. Diese epidemiologischen Daten weisen darauf hin, dass die Oberflächenthrombose und die tiefe Beinvenenthrombose bzw. Lungenembolie aus einem gemeinsamen $\mathrm{Zu}$ stand der Hyperkoagulabilität resultieren. Die Bedeutung der Hyperkoagulabilität wird auch dadurch unterstützt, dass die tiefe Beinvenenthrombose in bis zu $10 \%$ der Fälle am kontralateralen Bein nachzuweisen ist. Dementsprechend sind die Risikofaktoren wie Tumorleiden, chirurgische Eingriffe, Immobilität und Frakturen bei der Oberflächenthrombose dieselben wie bei der tiefen Beinvenenthrombose.

\section{Risikofaktoren für eine TVT als Folge einer OVT}

Bei Patienten mit akuten Oberflächenthrombosen können für die Zeit nach der akuten Krankheitsphase folgende Risikofaktoren für erneute thromboembolische Ereignisse identifiziert werden:

- Vorangegangene thromboembolische Ereignisse (tiefe Venenthrombosen, Lungenembolien, oberflächliche Venenthrombosen)
- Positive Familienanamnese tiefer Beinvenenthrombosen

- Oberflächenthrombose nicht in Varizen sondern in normalen Venen (5)

\section{Varikose als OVT Risikofaktor}

Als wesentlicher Risikofaktor der Oberflächenthrombose gelten Varizen. Die Relevanz der Varikose als Risikofaktor zeigt sich z. B. in der großen Häufigkeit von OVT-Rezidiven bei Patienten, bei denen die Varikose unbehandelt bleibt. In einer Studie bestätigte sich diese klinische Erfahrung bei Patienten mit einer Vorgeschichte einer spontanen Oberflächenthrombose und Varizen in einer Nachbeobachtungsperiode von im Mittel 55 Monaten (6). Die Patienten wurden bei ihrer primären Phase der Oberflächenthrombose alle täglich mit 2,5mg Fondaparinux über 45 Tage behandelt. Immerhin 13 der nachverfolgten 57 Patienten erlitten während der Nachuntersuchungsperiode eine erneute Oberflächenthrombose. Interessant war, dass zwar alle Rezidivpatienten einen Thrombophiliedefekt zeigten, es sich aber keine signifikanten Unterschiede zur Gruppe der Patienten ohne OVT-Rezidiv fanden.

\section{Wärme kein zusätzlicher Risikofaktor}

Man könnte denken, da bei Wärme Patienten mit Varizen nicht selten über zunehmende Schwellungen und Schweregefühle der Beine klagen, dass auch die Oberflächenthrombosen in der warmen Jahreszeit gehäuft auftreten würden. Dies konnte jedoch in einer großen Analyse mit $1395 \mathrm{~Pa}$ tienten mit einer Beobachtungszeit von 4,75 Jahren nicht belegt werden (7).

\section{D-Dimer Level in Varizen erhöht}

Bereits ohne klinisch manifeste Oberflächenthrombose sind in Varizen die D-Dimere als ein prothrombotischer Biomarker und ein sehr sensitives Maß für die endogene Fibrinolyse erhöht. Dies zeigt sich 
beim Vergleich der D-Dimer Level in Varizen im Vergleich zu den D-Dimer Level in den Armvenen desselben Patienten. Entnimmt man Patienten und gesunden Kontrollprobanden jeweils Blutproben aus einer gesunden Vene am Arm sowie einer Varize bzw. einer gesunden Vene am Unterschenkel, so ist der mediane D-Dimer Level in der Varize mit 319ng/ml signifikant höher als am Arm mit 218ng/ml $(p=0,003)(8)$. Demgegenüber unterscheiden sich die D-Dimer Level am Arm und am Unterschenkel in der gesunden Kontrollgruppe mit einem Median von 269ng/ $\mathrm{ml}$ versus $262 \mathrm{ng} / \mathrm{ml}$ nicht $(\mathrm{p}=0,361)$. Auch die Quotienten aus Unterschenkel- und Kubitalblut-D-Dimer Werten unterscheiden sich in der Patientengruppe mit 1,14 signifikant von der Kontrollgruppe mit 0,96 (8).

\section{Besonderes Krankheitsbild: Penile Mondor-Erkrankung}

Während insgesamt wie oben dargestellt der Begriff der Oberflächenthrombose zu bevorzugen ist, ist bei der Mondor-Erkrankung der Begriff der Thrombophlebitis noch am ehesten berechtigt. Die MondorErkrankung wurde 1939 primär für den lateralen Thorax, thorako-epigastrische oder die oberen epigastrischen Venen speziell bei Frauen beschrieben. Eine erste Beschreibung einer Phlebitis am Penis erfolgte 1955 durch Braun Falco im Rahmen einer generalisierten Phlebitis. 1958 wurde dann die isolierte Penisthrombophlebitis als penile Mondor-Erkrankung durch Helm und Hodge beschrieben (9). Entsprechend der späteren Beschreibung ist die penile Mondor-Erkrankung tatsächlich seltener als die thorakale Mondor-Erkrankung. Das klinische Bild am Penis ist typisch mit einer palpablen walzenartigen Struktur, welche mit einer Hautrötung einhergeht und druckschmerzhaft ist. Die Ätiologie der Erkrankung ist letztlich nicht geklärt. Ganz unterschiedliche Risikofaktoren werden genannt, wobei insbesondere Traumata eine wesentliche Rolle sowohl bei der thorakalen als auch bei der penilen Mondor-Erkrankung spielen ( $\triangleright$ Tab. 1).

Auch wenn für die Mondor-Erkrankung eine besonders starke entzündliche
Reaktion im Vergleich zur Thrombusgröße typisch zu sein scheint, lässt sich doch duplexsonographisch in der Regel eine nicht komprimierbare Vene erkennen (10).

\section{Cannabis und Oberflächen- thrombosen}

Lokale Faktoren wie die Varikose und systemische Einflussfaktoren wie eine allgemeine Hyperkoagulabilität können die Entstehung von Oberflächenthrombosen begünstigen. Wie viel Einfluss systemische Faktoren haben können, zeigte sich bei einem Cannabis-Raucher mit migratorischer Oberflächenthrombose (11). Der 28-jährige ansonsten gesunde Mann litt über $5 \mathrm{Jahr}$ rezidivierend an schmerzhaften Oberflächenthrombosen an beiden Beinen, aber auch am Fuß, der Hand und der Leiste. Risikofaktoren wie eine Thrombophilie, maligne Grunderkrankungen oder rheumatische Erkrankungen konnten ausgeschlossen werden. Erst nach einer intensiven Befragung stellte sich heraus, dass der Patient, der zuvor jeden Tabakgenuss verneint hatte, regelmäßig Cannabis rauchte. Dies tat er zum einen als Joint, angefertigt aus Zigarrenhüllen, bei denen der Tabak durch Cannabis ersetzt worden war, zum anderen aber auch als Cannabis-Tabak-Gemisch. Er hatte selber den Eindruck, dass sich vermehrt Oberflächenthrombosen ausbildeten, wenn er vermehrt Cannabis rauchte. Er hatte dann Phasen, in denen er reines Cannabis in einer Wasserpfeife rauchte, in

Tab. 1 Risikofaktoren für die penile Mondor Erkrankung

\begin{tabular}{l} 
- Exzessive und lange sexuelle Aktivität \\
- Überlange sexuelle Abstinenz \\
- Lokale oder auch entferntere Infektionen \\
- Vorgeschichte sexuell übertragbarer Erkran- \\
kungen \\
- Gebrauch intrakavernöser Medikament oder \\
Vakuumanwendung \\
- Thrombophilie \\
- Inguinale Hernienoperation \\
- Orchidopexie \\
- Morbus Behçet \\
- Bodybuilding Übungen \\
- Beckentumore \\
- Paraneoplastische Syndrome \\
- Intravenöser Drogenmissbrauch \\
- Allgemeine Thromboseneigung \\
\hline
\end{tabular}

denen die Frequenz und Intensität der Entzündungsperioden zurückgegangen seien. Nachdem dieser Zusammenhang thematisiert worden war, stellte der Patient dann für ein Jahr das Cannabisrauchen völlig ein und war komplett beschwerdefrei. Neue Oberflächenthrombosen traten erst wieder nach Wiederaufnahme des Cannabisgenusses auf. Auch wenn dieser Zusammenhang erst kasuistisch beschrieben worden ist, kann es sich bei ansonsten unklarer Genese einer Thrombophlebitis saltans doch lohnen, mit dem Patienten über einen möglichen Cannabiskonsum zu sprechen.

\section{Diagnostik der Oberflächenthrombose}

Eine Oberflächenthrombose kann in der Regel klinisch diagnostiziert werden. Trotzdem gilt die Duplexsonografie als unverzichtbares Hilfsmittel bei der Diagnose. Dabei sollten folgende Punkte berücksichtigt werden:

- Ausdehnung des Thrombus mit Thrombusanfang und Thrombusende

- Übergang in eine tiefe Beinvenenthrombose

- Untersuchung beider (!) Beine, da simultan mit einer Oberflächenthrombose nicht selten an der kontralateralen Gliedmaße eine tiefe Beinvenenthrombose auftritt

- Thrombuslänge über $5 \mathrm{~cm}$ in den Saphenavenen (Indikation zur Antikoagulation in Prophylaxe-Dosierung)

- Annäherung des Thrombus im Crossenbereich auf weniger als $3 \mathrm{~cm}$ an das tiefe Beinvenensystem heran (Indikation zur Antikoagulation in therapeutischer Dosierung)

Treten die Oberflächenthrombosen abseits von Varizen auf, sollten andere Risikofaktoren abgefragt werden. Hier sind insbesondere maligne Grunderkrankungen sowie andere Risikofaktoren für thromboembolische Ereignisse in Betracht zu ziehen (12). Ergänzend sollte anamnestisch und klinisch auch auf Zeichen der Lungenembolie geachtet werden. 


\section{Therapie der Oberflächen- thrombose}

Die Therapie der Oberflächenthrombose wird in der AWMF Leitlinie „Diagnostik und Therapie der Venenthrombose und der Lungenembolie“ vom 10.10.2015 definiert (13). Grundsätzlich soll die Indikation zu einer Antikoagulation geprüft werden. Bei einem transfaszialen Thrombuswachstum soll wie bei einer Venenthrombose vorgegangen werden. Die Behandlung der Oberflächenthrombose hängt von der Art des betroffenen Gefäßes und von der Thrombusausdehnung ab ( Tab. 2).

Oberflächenthrombosen in einem varikös degenerierten Gefäß sollten zum Anlass für die Sanierung der Varikose genommen werden. Dabei ist die Komplikationsrate bei zunächst konservativer Therapie und nachfolgender Sanierung im beschwerdefreien Intervall geringer als bei sofortiger Operation während noch bestehender florider Oberflächenthrombose. Ggf. kann eine sofortige operative Sanierung von OVT und Varikose in Erwägung gezogen werden, wenn es sich um ein sehr frisches thrombotisches Geschehen handelt. In der Leitlinie wird begleitend zur Antikoagulation eine Kompressionsbehandlung bis zum Abklingen der Symptome, in der Regel über drei Monate empfohlen.

\section{Wie kosteneffektiv ist die derzeitige Therapie der Oberflächenthrombose?}

Die in der deutschen Leitlinie zur Thrombose und Lungenembolie festgehaltenen Therapieprinzipien werden international angewendet und sind fest etabliert. Trotzdem gibt es kritische Diskussionen in Bezug auf die Empfehlung einer mehrwöchigen Antikoagulation in prophylaktischer Dosierung bei Oberflächenthrombosen der Saphenavenen. Diese Empfehlung stützt sich auf die sogenannte CALISTO-Studie. Hier konnte ein Vorteil der Therapie mit Fondaparinux über 45 Tage gegenüber Placebo belegt werden. Entsprechend ihrem Stellenwert und der Qualität der Studie ist sie hochrangig im New England Journal of Medicine publiziert (14). Allerdings wird
Tab. 2 Art der Behandlung der Oberflächenthrombose

\begin{tabular}{l|l}
\hline Betroffene Vene & Therapie \\
\hline Kleinkalibrige Astvarizen/Seitenäste & $\begin{array}{l}\text { Kühlung, Kompressionstherapie, nicht steroidale An- } \\
\text { tiphlogistika bei Bedarf, Stichinzision mit Thrombus- } \\
\text { expression }\end{array}$ \\
\hline $\begin{array}{l}\text { V. saphena magna oder parva oder großka- } \\
\text { librige Varizenäste ab einer Thrombuslänge } \\
\text { von } 5 \mathrm{~cm}\end{array}$ & $\begin{array}{l}\text { Fondaparinux in prophylaktischer Dosierung }(2,5 \mathrm{mg} / \\
\text { Tag s.c.) über mindestens } 4 \text { Wochen + Kompressions- } \\
\text { therapie über } 3 \text { Monate }\end{array}$ \\
\hline $\begin{array}{l}\text { Thrombus näher als 3cm von einer Mün- } \\
\text { dungsklappe zum tiefen Venensystem ent- } \\
\text { fernt bzw. bei Übergreifen in das tiefe Ve- } \\
\text { nensystem }\end{array}$ & $\begin{array}{l}\text { Antikoagulation wie bei einer tiefen Venenthrombose } \\
\text { in therapeutischer Dosis über mindestens } 3 \text { Monate } \\
+ \text { Kompressionstherapie über mindestens } 6 \text { Monate }\end{array}$ \\
\hline
\end{tabular}

von Kritikern angeführt, dass auch in der Placebogruppe nur eine sehr geringe Rate von Thrombosen und Lungenembolien aufgetreten sei, die bei lediglich 1,3\% lag. Dies bedeutet, dass 98,7\% der Patienten auch ohne medikamentöse Behandlung keine Thrombose oder Lungenembolie bekommen. Wünschenswert erscheint daher eine bessere Selektion der Patienten, die wirklich einer Behandlung bedürfen (15).

\section{Rivaroxaban bei Oberflächenthrombosen}

In der sogenannten SURPRISE-Studie wurde Rivaroxaban in einer Dosierung von 10mg mit Fondaparinux in einer Dosierung von $2,5 \mathrm{mg}$ in täglicher Dosis über 45 Tage in der Therapie der Oberflächenthrombose unter dem Aspekt der Verhütung thromboembolischer Komplikationen verglichen. Um dem Vorwurf der begrenzten Kosteneffektivität der CALISTO-Studie $\mathrm{zu}$ begegnen, wurde in der SURPRISE-Studie ein zusätzliches Risikoprofil der Patienten gefordert (16). In die Studie konnten nur Patienten eingeschlossen werden, die mindestens einen der folgenden Risikofaktoren aufwiesen: Alter über 65 Jahre, männliches Geschlecht, Oberflächenthrombose oder tiefe Beinvenenthrombose oder Lungenembolie in der Vorgeschichte, Krebsleiden aktuell oder in der Vorgeschichte, Autoimmunerkrankung oder nicht variköse Venen betroffen. Wie in der CALISTO-Studie auch wurde die Behandlungsdauer auf 45 Tage angesetzt. Bemerkenswerterweise trat der wesentliche Anteil der thromboembolischen Komplikationen in der SURPRISE-Studie anders als in der
CALISTO-Studie erst nach Beendigung der 45tägigen Therapie auf. Obwohl die Patienten in der SURPRISE-Studie alle mindestens einen der oben genannten Risikofaktoren aufwiesen, stieg die Zahl thromboembolischer Komplikationen nicht so an wie ursprünglich angenommen. Anders als kalkuliert traten in der Thera$1,8 \%$ thromboembolische Ereignisse auf, so dass gefragt werden muss, wie sicher die Statistik zur Nicht-Unterlegenheit ist. Letztlich war weder die Zahl der thromboembolischen Ereignisse noch die Zahl der non-major Blutungen signifikant unterschiedlich, obwohl in der RivaroxabanGruppe thromboembolische Ereignisse bei $3,32 \%$ gegenüber $1,79 \%$ in der Fondaparinux-Gruppe auftraten und non-major Blutungen in der Rivaroxaban-Gruppe bei $2,54 \%$ der Patienten auftraten, aber nur bei 0,43\% der Fondaparinux-Gruppe. Unterwartet war auch, dass in der oralen Rivaroxaban-Gruppe mehr Patienten wegen fehlender Applikation der Medikation aus der Studie ausgeschlossen werden mussten als in der Fondaparinux-Gruppe (Rivaroxaban 10 Ausschlüsse, Fondaparinux 2 Ausschlüsse).

Was bedeuten nun die Ergebnisse der SURPRISE-Studie für die tägliche Praxis? Patienten mit den o.g. Risikofaktoren haben ein gesteigertes Risiko, auch nach einer 45-tägigen Antikoagulation in Prophylaxedosierung thromboembolische Komplikationen zu entwickeln. Derzeit ist unklar, ob eine Verlängerung der prophylaktischen Antikoagulation über 45 Tage hinaus oder der Wechsel von einer prophylaktischen zu einer therapeutischen Antikoagulation sinnvoll wäre. Zumindest für den definierpiegruppe nicht bei $3 \%$ sondern nur bei 
ten Applikationszeitraum von 4-6 Wochen muss der Zusammenhang der Applikationsform und der Adhärenz überprüft werden, da die Adhärenz in der SURPRISEStudie in der Gruppe mit oraler Medikation schlechter war als in der Gruppe mit subkutaner Applikation.

\section{Zeitverlauf und Rekanalisa- tionsrate nach Oberflächenthrombosen}

Duplexsonographische Kontrollen bei Oberflächenthrombosen dienen insbesondere dem Nachweis einer möglichen Progredienz des Thrombus in das tiefe Venensystem oder auch nur eine Verlängerung der Thrombuslänge im oberflächlichen Venensystem. Nicht selten wünschen Patienten die Kontrolle, ob bereits eine Rekanalisation stattgefunden hat, insbesondere auch zum Ende der medikamentösen Behandlung nach 4-6 Wochen. Dabei ist es wichtig zu wissen, dass die duplexsonographisch bestimmte Thrombusdicke und Thrombuslänge in den ersten 6 Wochen auch unter Therapie in der Regel sich kaum verändert. Demgegenüber bilden sich die klinischen Entzündungszeichen wie Rötungsschmerz und Überwärmung innerhalb von 6 Wochen regelmäßig zurück. Ein deutlicher Rückgang der Thrombuslänge und des Thrombusdurchmessers zeigt sich erst in den Kontrollen nach 3 und 6 Monaten (17). Demnach scheint es sinnvoll zu sein, den Patienten schon zu Beginn der Untersuchung darauf hinzuweisen, dass auch nach 6 Monaten mit einem thrombotischen (Teil-)Verschluss des betroffenen Gefäßes zu rechnen ist. Dies bedeutet nicht, dass eine weitere medikamentöse Behandlung erforderlich ist.
Interessenkonflikt

Nach Angaben der Autoren bestehen keine Interessenkonflikte.

\section{Ethische Richtlinien}

Für das Manuskript wurden keine Studien an Menschen oder Tieren durchgeführt.

\section{Literatur}

1. Di Minno MN, Ambrosino P, Ambrosini F, Tremoli E, Di Minno G, Dentali F. Prevalence of deep vein thrombosis and pulmonary embolism in patients with superficial vein thrombosis: a systematic review and meta-analysis. J Thromb Haemost. 2016 May;14(5):964-72. doi: 10.1111/jth.13279. Epub 2016 Mar 15.

2. Dua A, Heller J, Patel B, Desai S. Variability in the Management of Superficial Venous Thrombophlebitis across Practitioners Based in North America and the Global Community. Thrombosis. 2014;2014:306018. doi: 10.1155/2014/306018. Epub 2014 Oct 12.

3. Frappé P, Buchmuller-Cordier A, Bertoletti L, Bonithon-Kopp C, Couzan S, Lafond P, Leizorovicz A, Merah A, Presles E, Preynat P, Tardy B, Décousus H; STEPH Study Group. Annual diagnosis rate of superficial vein thrombosis of the lower limbs: the STEPH community-based study. J Thromb Haemost 2014; 12(6): 831-838.

4. Cannegieter SC, Horváth-Puhó E, Schmidt M, Dekkers OM, Pedersen L, Vandenbroucke JP, Sørensen HT. Risk of venous and arterial thrombotic events in patients diagnosed with superficial vein thrombosis: a nationwide cohort study. Blood 2015; 125(2): 229-235

5. Cosmi B, Filippini M, Campana F, Avruscio G, Ghirarduzzi A, Bucherini E, Camporese G, Imberti D, Legnani C, Palareti G; Risk factors for recurrent events in subjects with superficial vein thrombosis in the randomizes clinical trail SteFlux (Superficial Thromboembolism Fluxum). Thromb Res 2014; 133(2): 196-202.

6. Karathanos C, Spanos K, Saleptsis V, Tsezou A, Kyriakou D, Giannoukas AD. Recurrence of superficial vein thrombosis in patients with varicose veins. Phlebology 2015 Jul 16.
7. Frappé P, Bertoletti L, Presles E, Buchmuller-Cordier A, Merah A, Le Hello C, Peycelon D, Tardy B, Décousus $H$. Seasonal variation in the superficial vein thrombosis frequency. Thromb Res. 2015 Dec;136(6):1116-1119.

8. Lattimer CR, Kalodiki E, Geroulakos G, Syed D, Hoppensteadt D, Fareed J. d-Dimer Levels are Significantly Increased in Blood Taken From Varicose Veins Compared With Antecubital Blood From the Same Patient. Angiology 2015 Oct; 66(9): 882-888.

9. Kantarcı UH, Dirik A, Öztürk YE, Kiriș İ, Duymuş M. Doppler Ultrasound and Magnetic Resonance Imaging Findings of Penile Mondor's Disease: Case Report and Literature Review. Pol J Radiol. 2016 Feb 2;81:36-8. doi: 10.12659/PJR.895569. eCollection 2016

10. Marsaudon E, Legal C, Gayoux D, Weber O. La maladie de Mondor pénienne: une observation. Rev Med Interne (2016).

11. Lee C, Moll S. Migratory superficial thrombophlebitis in a cannabis smoker. Circulation 2014; 130(2): 214-215

12. Sándor T. [Superficial venous thrombosis. A state of art]. Orv Hetil. 2017 Jan;158(4):129-138.

13. AWMF Leitlinie: Diagnostik und Therapie der Venenthrombose und der Lungenembolie. Verabschiedet am 10. Oktober 2015.

14. Decousus H, Prandoni P, Mismetti P, Bauersachs RM, Boda Z, Brenner B, Laporte S, Matyas L, Middeldorp S, Sokurenko G, Leizorovicz A; CALISTO Study Group. Fondaparinux for the treatment of superficial-vein thrombosis in the legs. N Engl J Med 2010 Sep 23; 363(13): 1222-1232.

15. Blondon M, Righini M, Bounameaux H, Veenstra DL. Fondaparinux for isolated superficial vein thrombosis of the legs: a cost-effectiveness analysis. Chest. 2012 Feb;141(2):321-329.

16. Beyer-Westendorf J, Schellong SM, Gerlach H, Rabe E, Weitz JI, Jersemann K, Sahin K, Bauersachs R; SURPRISE investigators. Prevention of thromboembolic complications in patients with superficial-vein thrombosis given rivaroxaban or fondaparinux: the open-label, randomised, noninferiority SURPRISE phase $3 \mathrm{~b}$ trial. Lancet Haematol. 2017 Mar;4(3):e105-e113.

17. Spirkoska A, Jezovnik MK, Poredos P.Time Course and the Recanalization Rate of Superficial Vein Thrombosis Treated With Low-Molecular-Weight Heparin. Angiology. 2014 May 7. [Epub ahead of print] 